\title{
DEVELOPMENT OF MUSES-ACR ELECTRON COOLER
}

\author{
T. Tanabe ${ }^{\#}$, T. Rizawa ${ }^{+}$, K. Ohtomo ${ }^{*}$, and T. Katayama, RIKEN, Saitama, JAPAN
}

\section{Abstract}

An electron cooler (EC) device for the Accumulator Cooler Ring (ACR) in the MUSES project [1] is currently under development. The electron beam energy is varied from $30 \mathrm{keV}$ to $250 \mathrm{keV}$ with a maximum current of 4.1 A. An axial magnetic field of $0.2 \mathrm{~T}$ in a $3.6 \mathrm{~m}$ solenoid is chosen to satisfy the magnetization condition for the lightest ions and the expansion factor of 20 corresponds to the field of $4 \mathrm{~T}$ in the gun section. The details of the design and technological issues are discussed.

\section{INTRODUCTION}

The design of the ACR-EC has been modified since it was first reported at EPAC98 [2] due to various reasons. One of the main concerns is the vacuum level near the gun cathode which is to be operated under severe conditions. The maximum acceleration voltage has been reduced from 300 to $250 \mathrm{kV}$, and the maximum magnetic field at the gun section has been decreased from 5 to $4 \mathrm{~T}$. The collector is partitially based upon TARN-II collector [3] with a number of modifications. The design of the gun section is described in Sec. 2 and that of the collector is given in Sec. 3. Effects of the troidal section on the electron beam are examined in the following section before discussion.

\section{GUN SECTION}

\subsection{Cathode/Anode and Acceleration Tube}

The initial design of the gun had a magnetic field of $5 \mathrm{~T}$ and acceleration voltage of $300 \mathrm{kV}$. After careful examination of the design, we have concluded that the reduction of expansion factor from 25 to 20 gives only small degradation of the performance in terms of lowest achievable electron transverse energy [4]. The maximum nominal energy of the injected ion beam from the SRC ring is $400 \mathrm{MeV} / \mathrm{u}$ which corresponds to the electron kinetic energy of $219.4 \mathrm{keV}$. Therefore, $250 \mathrm{kV}$ is deemed to be sufficient.

The cathode diameter is increased from 10 to $12.7 \mathrm{~mm}$ in order to reduce the maximum voltage gradient in the vicinity of the cathode/anode from 50 to $36 \mathrm{kV} / \mathrm{cm}$, while maintaining the same anode/cathode voltage $(30 \mathrm{kV})$ as before.

\footnotetext{
"Email: ttanabe@ postman.riken.go.jp

${ }^{+}$On leave from Toshiba corporation. *On leave from Sumitomo Heavy Industries.
}

The maximum emision current density is also reduced from 5.1 to $3.15 \mathrm{~A} / \mathrm{cm}^{2}$. We plan to use an Ir-coated dispenser cathode produced by Toshiba Co. At one point, a fair amount of effort was made to reduce the diameter of the accelerator tube to make the bore size of a superconducting solenoid smaller. However, it turns out that the vacuum level near the cathode could never be safisfactory due to poor vacuum conductance of the small apertures of diaphrams in the original design $(26 \mathrm{~mm})$. This estimate assumes the outgassing rate from cold surfaces, so it would be worse under more realistic circumstances. Hence, the idea of small tube was abondoned for larger vacuum conductance. Figure 1 shows a result of EGUN simulation with the current design.

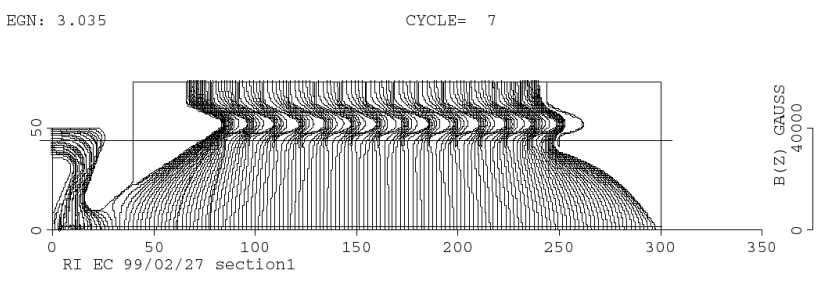

Fig. 1: EGUN simulation of the electron gun section

\subsection{Magnetic Shielding}

The design of the magnetic shielding at the gun section was optimized by SAM [5]. A schematic picture of the configuration of a superconducting solenoid, a warm solenoid and a correction coil and a magnetic shielding is delineated in Fig2. The magnetic field distribution and the adiabatic parameter is shown in Fig. 3 .

(3)

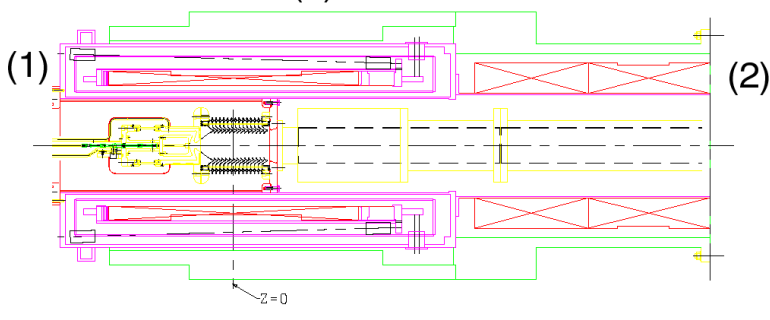

Fig. 2: Layout of (1) a superconducting solenoid, (2) a warm solenoid and (3) a magnetic shielding. 


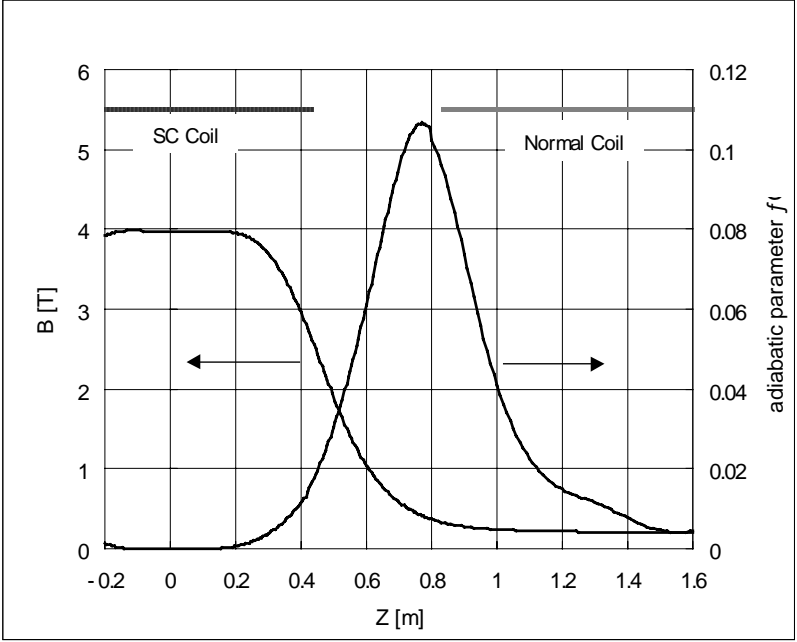

Fig. 3: The longitudinal magnetic distribution and the adiabatic parameter.

\section{COLLECTOR SECTION}

The initial design of the ACR collector was based upon the TARN II collector. Various improvement has been incorporated during the past year. The geometry of the new collector section is given in the Fig. 4. The collector consists of a deceleration tube, a repeller electrode, a collector electrode and two permanent magnetic coils. The collector electrode is a Faraday cap.

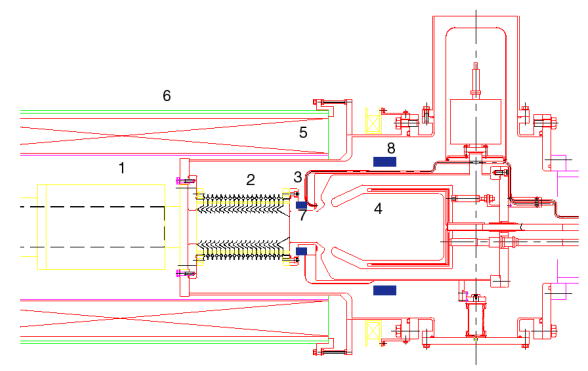

Fig. 4: The design of the collector. 1 is the drift chamber, 2 deceleration tube, 3 repeller, 4 collector electrode, 5 the solenoid of electron cooling, 6 magnetic shielding, 7 a permanent magnet coil placed on the collector entrance, and 8 is a permanent magnet coil placed on the collector surface.

The ratio of the current density of the reflected secondary electrons that escape a collector to that of the incoming beam is proportional to [6];

$$
\frac{j_{r e f}}{j} \cong \frac{\sigma_{e}}{\pi}\left(\frac{\varphi_{\text {min }}}{U_{c o l}}\right)^{2} \frac{B_{c o l}}{B_{\varphi \min }},
$$

where $\mathrm{j}$ is the incoming beam current, $\sigma_{e} \approx 1$ is the secondary emission coefficient, $\mathrm{B}_{\text {col }}$ is the magnetic field on the collector surface, and $\mathrm{B}_{\varphi_{\min }}$ is the magnetic field in the region of minimum potential value.

Types of modifications applied are as follows:

- The collector diameter is increased to reduce the magnetic field on the collector surface, $\mathrm{B}_{\mathrm{col}}$.

- To increase $B_{\varphi_{\min }}$, the permanent magnet coil is placed inside the repeller electrode with the magnetic field of $2 \mathrm{kG}$.

- A permanent magnet coil is placed on the collector surface to reduce $\mathrm{B}_{\text {coll }}$.

- The collector radius is reduced at the entrance to shift the position of the virtual cathode to the region in higher magnetic field.

The collector perveance is estimated as $\mathrm{P}_{\text {col }}=\mathrm{I} / \mathrm{U}_{\text {col }}{ }^{3 / 2} \approx$ $25 \sim 30 \mu \mathrm{A} / \mathrm{V}^{3 / 2}$. As a result, the current loss was reduced by an order of magnitude compared to the old design.

\section{TORIODAL SECTION}

The electrons obtain additional perturbation, when they pass through the EC toroidal section. This perturbation is observed for electrons which are displaced from the equilibrium axial trajectory. The guiding longitudinal magnetic field in toroidal section depends on the radial co-ordinate $x$.

$B=B /(1+x / R)$

where $\mathrm{R}$ is the radius of the axial electron trajectory in the toroidal section. The transverse bending magnetic field used for compensation of the electron drift motion in the toroidal magnet is equal to

$B_{y}=p / e R$,

where $p$ is the electron momentum. The drift motion caused by the centrifugal force $F_{s}=m m v^{2} /(R+x)$ is not entirely compensated by the transverse bending magnetic field $\mathrm{B}_{\mathrm{y}}$ for the electrons whose trajectories are shifted from beam axis on the distance $x$. As a result, when the electrons pass through toroidal magnet, the additional drift velocity appears;

$v_{d T}=\frac{m v^{2}}{\operatorname{Re} B} \frac{x}{R}=v \frac{\rho}{R} \frac{x}{R}$,

where $\rho=v / \omega_{B}$ is the larmor radius. The transverse temperature corresponding to this drift velocity is equal to

$T_{d T}=\frac{m v_{d T}^{2}}{2}=\frac{m v^{2}}{2}\left(\frac{\rho}{R} \frac{x}{R}\right)^{2}$. 
Discontinuity of the magnetic field at the entrance/exit of toroidal magnets significantly increase the transverse temperature. This increase strongly depends on the radius of curvature, $\mathrm{R}$ as is shown in Eq. (5). It has been increased from 1.2 to $1.5 \mathrm{~m}$ to make this contribution comparable to the initial temperature even in case of abrupt field change.

\section{DISCUSSION}

Table 1 shows the latest parameters of the device and cross-sectional view of the whole EC system is shown in Fig. 5.

Table 1: Parameters of ACR-EC

\begin{tabular}{|l|l|}
\hline Acceleration Voltage & $30 \sim 250 \mathrm{kV}$ \\
\hline Magnetic Field (gun/cooling) & $4 \mathrm{~T} / 0.2 \mathrm{~T}$ \\
\hline Field Uniformity (gun/cooling) & $2 \times 10^{-3} / 5 \times 10^{-5}$ \\
\hline Cathode Diameter & $12.7 \mathrm{~mm}$ \\
\hline Maximum E-Beam Current & $4 \mathrm{~A}$ \\
\hline Gun Perveance & $0.79 \mu \mathrm{P}$ \\
\hline Anode-Cathode Voltage & $30 \mathrm{kV}$ \\
\hline Main Solenoid length & $3.6 \mathrm{~m}$ \\
\hline Toroidal Angle / Radius & $90^{\circ} / 1.5 \mathrm{~m}$ \\
\hline Collector Efficiency & $>99.98 \%$ \\
\hline
\end{tabular}

In the initial plan, the EC is supposed be installed in one of the short straight sections in the ACR. However, insertion of correction solenoids in the adjecent cells lead to unnecessary complexion in terms of beam dynamics [7]. Therefore, the device is likely to be installed in one of long straight section in a modified lattice of ACR. The modification is currently underway.

\section{ACKNOWLEDGMENTS}

The authors wish to thank Dr. Evgeny Syresin and Dr. Igor Meshkov of Joint Institute of Nuclear Research in Russia for their advice, and Mr. Akira Yamashita of Toshiba Co. for his asistance.

\section{REFERENCES}

[1] T. Katayama, et al., "MUSES Project of RIKEN RI Beam Facotry", p. 529, proceedings of EPAC'98.

[2] I. Watanabe, et al., "Electron Cooling at ACR in MUSES Project", p. 2258, proceedings of EPAC'98.

[3] T.Tanabe, et al., "Electron Cooling Experiments at INS", NIM A 307 (1991), p.7.

[4] E. Syresin, et al., "Simulation and Design of an Electron Cooling System for ACR," RIKEN internal report.

[5] M. Tiunov, et al., Preprint INP, Novosibirsk, pp. 85-159.

[6] E. Syresin, "The Parameters of the Secodary Electrons in the Electron Cooling System", NIM A, 391 (1997), p. 114.

[7] T. Tanabe, et al., "Development of ACR Electron Cooler," RIKEN Accel. Prog. Rep. (1998), to be published.

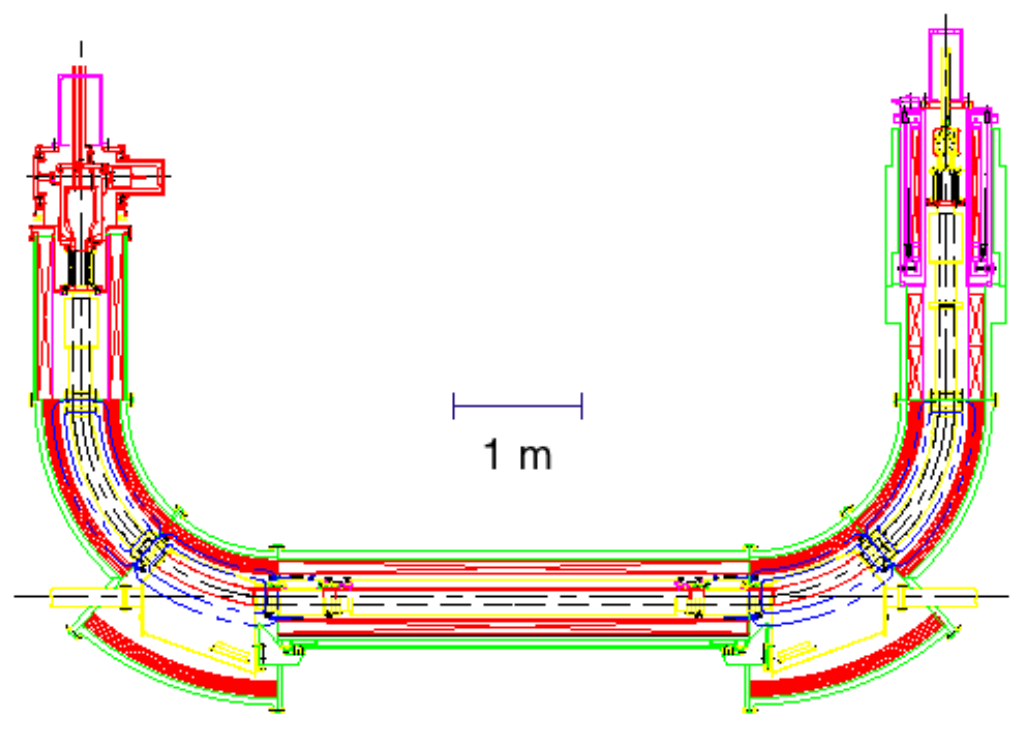

Fig. 5 Cross-sectional view of the MUSES-ACR EC 